\title{
Produções científicas e regulamentações sobre qualidade em diálise no Brasil: uma revisão integrativa
}

\author{
Scientific production and quality regulations on \\ dialysis in Brazil: an integrative review
}

RESUMO A presente pesquisa teve como objetivos: conhecer as produções científicas e regulamentações sobre qualidade em diálise no Brasil; identificar as regulamentações que subsidiam a qualidade nesses serviços; e classificá-las conforme o modelo de avaliação da qualidade do Avedis Donabedian. Trata-se de uma revisão integrativa da literatura na qual os critérios de inclusão foram artigos originais e regulamentações que abordam a qualidade nos serviços de diálise no Brasil, publicados em português e inglês, no período de 1996 a 2017. Foram analisados 13 artigos e quatro regulamentações. Os dados foram classificados à luz da tríade Donabediana de avaliação da qualidade: Estrutura, Processo e Resultado. Foi verificado que $50 \%$ dos artigos avaliam o componente estrutura, enquanto que o componente processo e resultado estiveram presentes em 7\% dos estudos, e estrutura e resultado estiveram em 14\% dos artigos. Não houve artigo que avaliasse as três dimensões simultaneamente. Foi constatado que a maioria dos estudos se preocupa em estudar as adequações referentes às portarias que estabelecem recursos mínimos para o funcionamento do serviço de diálise. Faz-se necessário compreender que a estrutura adequada é um fator subsidiador da qualidade, mas é por meio da relação estabelecida no processo que os resultados são delimitados, referenciando a intersecção das três dimensões.

Palavras-Chave: Qualidade da Assistência À Saúde. Avaliação de Serviços de Saúde. Diálise. Nefrologia.

Aвstract The objective of this study is to know the scientific productions and regulations about the quality of Dialysis services in Brazil; to identify the regulations that subsidize quality in dialysis services in Brazil; and classify them according to the Avedis Donabedian quality assessment model. It is an integrative review of the literature and the inclusion criteria were original articles and regulations that address the quality of Dialysis services in Brazil from 1996 to 2017, published in Portuguese and English. 13 articles and 4 regulations were analyzed. The data was categorized using the triad of the Donabedian model of evaluation: Structure; Process and Outcome. Process and Result. It was verified that $50 \%$ of the articles evaluated only the structure component, and the component process and result were present in $7 \%$ of the studies, already structure and result were in $14 \%$ of the articles. There was no article evaluating the three categories. It was contacted that most studies are concerned with studying the appropriateness of regulatory institutions,

Darlene Santos Almeida Universidade do Estado da Bahia (UNEB), Salvador/BH - Brasil. Edenise Maria Santos da Silva BATALHA

Universidade do Estado da Bahia (UNEB), Salvador/BH - Brasil. which establish minimum resources for the functioning of institutions. It is necessary to understand that the proper structure is a factor that supports quality, but it is through the relation established in the process that the results are delimited, referring to the intersection of the three dimensions.

Key-words: Quality of Health Care. Evaluation of Health Services. Dialysis. Nephrology. 


\section{INTRODUÇÃO}

Cuidar deriva do latim cogitare, que significa "imaginar", "pensar", "meditar", "julgar", "tratar", "aplicar" a atenção, "refletir", "prevenir" e ter-se, cuidar é o "cuidado" em ato. $\mathrm{O}$ cuidado em saúde é a ação integral da assistência, na qual o indivíduo cuidado é ativo em todos esses processos, que incluem o acolhimento humanizado, o atendimento integral, a recuperação segura e o tratamento digno. ${ }^{1}$

Nesse contexto do cuidado, a qualidade em saúde, segundo o Modelo Donabediano, traduz-se como o desenvolvimento do saber/fazer profissional com os recursos disponíveis oferecendo menor risco para o paciente. $\mathrm{O}$ citado modelo avalia qualidade em três dimensões: 1. Estrutura como a composição necessária para a execução do processo assistencial; 2 . Processo que corresponde à prestação da assistência de acordo com os padrões técnicos e científicos e, portanto, a relação entre o profissional e o paciente; e o 3. Resultado, este torna-se consequência de efeito qualitativo para o paciente e para o profissional que oferece o cuidado, alcançando o principal objetivo: melhora do paciente e a satisfação do profissional. ${ }^{2}$

Esta tríade "Estrutura, processo e resultado" proposta por Avedis Donabedian como modelo de avaliação da qualidade em saúde, inspirada na teoria sistêmica, ressalta que só faria sentido se houvesse relação de causalidade entre estrutura, processo e resultados de determinada realidade. ${ }^{3}$

Cada componente da tríade tem valor e significado diferentes, a seguir no Quadro 1. O conceito de cada componente:
QUADRO 1. Conceituação das dimensões estrutura, processo e resultado, segundo modelo de avaliação da qualidade de Donabedian Avedis. ${ }^{4}$

\begin{tabular}{|l|l|}
\hline Componente & Conceito \\
\hline Estrutura & $\begin{array}{l}\text { Envolve os recursos humanos, } \\
\text { físicos, materiais e financeiros, } \\
\text { os equipamentos, as políticas } \\
\text { de educação permanente } \\
\text { e os estabelecimentos de } \\
\text { protocolos assistenciais. }\end{array}$ \\
\hline Processo & $\begin{array}{l}\text { Corresponde ao conjunto } \\
\text { de atividades na produção, e } \\
\text { no setor saúde, nas relações } \\
\text { estabelecidas entre os } \\
\text { profissionais e os usuários, } \\
\text { incluindo busca pelo } \\
\text { diagnóstico e pela terapêutica } \\
\text { empregada. }\end{array}$ \\
\hline Resultado & $\begin{array}{l}\text { Consiste na obtenção das } \\
\text { características desejáveis } \\
\text { dos produtos ou serviços, } \\
\text { retratando os efeitos da } \\
\text { assistência à saúde do } \\
\text { paciente e da população, } \\
\text { o custo mensurado e se } \\
\text { esse é compatível com } \\
\text { a sustentabilidade da } \\
\text { organização. }\end{array}$ \\
\hline
\end{tabular}

Fonte: DONABEDIAN, A. The definition of quality and approaches to its assessment: explorations in quality assessment and monitoring. Health Administration Press, Chicago (US), 1988; 260 (12), p. 1.743-8.

Esse modelo tem apresentado grande aceitabilidade na área de avaliação da qualidade em saúde e, por esse motivo, tem sido amplamente difundido com o objetivo de exercer vigilância contínua da qualidade, detecção e correções precoce de alterações de desvios dos padrões. ${ }^{5}$ 
Dessa forma, a qualidade na assistência está intimamente ligada ao cuidado que se dá ao paciente. Hoje, é comum a mídia explicitar as filas para atendimento público brasileiro, a falta de estrutura física, de recursos humanos e materiais para desenvolver um trabalho de qualidade. No entanto, o Ministério da Saúde vem tentando mudar esse cenário, incentivando a acreditação hospitalar, como também outros meios de avaliação e melhora da qualidade, no intuito de estabelecer atendimento humanizado, tendo em sua estrutura física, tecnológica, humana e administrativa, valores que buscam o respeito do ser humano, colocando-se a serviço deste, garantindo-lhe um atendimento de elevada qualidade. ${ }^{6}$

No que tange ao atendimento de diálise, um episódio importante trouxe para essa área novos direcionamentos para a qualidade da assistência. Em fevereiro de 1996, em Pernambuco, uma tragédia aconteceu numa clínica de hemodiálise na cidade de Caruaru. Pacientes renais crônicos que eram submetidos ao tratamento de hemodiálise apresentaram quadro clínico de cefaleia, tonturas, distúrbio visual e astenia. Uma pesquisadora do Departamento de Biofísica da Universidade Federal do Rio de Janeiro levantou a hipótese de intoxicação por toxinas provenientes de algas cianofíceas. No dia 11 de abril de 1996, a Secretaria Estadual de Saúde de Pernambuco publicou oficialmente, como causa das mortes: “...uma intoxicação pela água usada no tratamento que estava contaminada com a toxina microsistina-LR".

Dos 142 pacientes que foram expostos à toxina, 50\% evoluíram a óbito. Infelizmente, foi preciso uma catástrofe com repercussão nacional para que os membros participantes da Gestão Integrada do Meio Ambiente e dos Recursos Hídricos e da Vigilância da Saúde repensassem na metodologia e na formulação de padrões de qualidade tanto do tratamento de água para o abastecimento público, quanto para o tratamento dialítico. ${ }^{7}$

Diante disso, entendendo a importância de se abordar a temática qualidade e sua avaliação em serviços de diálise é que este estudo foi desenvolvido, tendo como pergunta norteadora: Quais as características das produções científicas e regulamentações que abordam a qualidade nos serviços de diálise no Brasil? Para responder à referida pergunta, foram traçados os seguintes objetivos: Conhecer as produções científicas que abordam a qualidade em serviços de diálise no Brasil; Identificar as regulamentações que subsidiam a qualidade nos serviços de diálise no Brasil; e Classificar as produções científicas e regulamentações conforme o modelo de avaliação da qualidade do Avedis Donabedian.

A relevância desta pesquisa traduz-se na possibilidade de ser subsídio para a melhoria da qualidade nos serviços de diálise. Ainda, visa compartilhar a temática com a comunidade científica, sendo uma forma de abordar as lacunas existentes sobre o tema.

\section{Metodologia}

Trata-se de uma revisão integrativa da literatura desenvolvida por meio dos seguintes passos: elaboração da pergunta/busca ou amostragem na literatura /Categorização dos estudos/análise crítica dos dados incluídos /discussão dos resultados/Síntese do conhecimento. ${ }^{8}$ 
Os critérios de inclusão foram artigos originais que abordam a qualidade nos serviços de diálise no Brasil publicados em português e inglês e que estivessem disponíveis na íntegra. O período para o início da coleta se deu a partir da $1^{\text {a }}$. portaria $n^{\mathrm{o}} ; 2.042$, de 11 de outubro de 1996 que estabeleceu o Regulamento Técnico para Funcionamento dos Serviços de Terapia Renal Substitutiva e define as exigências mínimas para o funcionamento das Unidades de Diálise e Unidades de Transplante Renal. ${ }^{9}$ Os critérios de exclusão foram artigos na modalidade revisão de literatura e editoriais.

Foi realizada consulta na base de dados Biblioteca Virtual em Saúde-BVS utilizando os seguintes descritores de pesquisa combinados com o booleano "AND": 1. "Garantia da qualidade dos cuidados de saúde" and "Diálise"; 2o. "Controle de Qualidade" and "Diálise" 3\%. "Qualidade da Assistência em Saúde" and "Diálise"; 4. "Qualidade Acesso e Avaliação da Assistência em Saúde" and "Diálise"; 50. "Enfermagem em Nefrologia" and "Qualidade"; 60. "Indicadores de Qualidade na Saúde" and "Diálise"; 7. "Indicadores de Qualidade em Assistência à Saúde" and "Diálise" 8. "Qualidade de Água" and "Diálise" 9. "Indicadores de Qualidade" and "Nefrologia"; $10^{\circ}$. "Indicadores de qualidade" and "Acesso de Hemodiálise"; 11. "Equipe de Qualidade" and "Diálise"; 12. "Instrumento para Avaliar Qualidade" and "Diálise" 13․ "Instrumentos para avaliar qualidade de assistência" and "Diálise"; 14. "Qualidade" and "Reuso and "diálise".

$\mathrm{Na} 1^{\mathrm{a}}$. fase de avaliação dos dados: As combinações resultaram num total de 912 artigos. Foi realizada a leitura dos títulos, resumo e palavras-chave. $\mathrm{Na} 2^{\text {a }}$. fase foi fei- ta a seleção dos artigos aplicando os critérios de exclusão, foram excluídos 864 por não se enquadrarem no objetivo do estudo e sete artigos que contemplavam o censo da Sociedade Brasileira de Nefrologia-SBN por apresentar apenas dados estatísticos e 28 artigos foram excluídos por se repetirem durante a pesquisa, finalizando a pesquisa com 13 artigos.

A busca das regulamentações sobre qualidade de diálise no Brasil foi realizada nas fontes: Agência Nacional de Vigilância Sanitária (ANVISA), Ministério da Saúde (MS) e na Sociedade Brasileira de Nefrologia $(\mathrm{SBN})$. Entre recomendações, nota técnica e portarias do Ministério da Saúde, foram encontradas 15 publicações, foram excluídas 11 por não alcançarem o objetivo da pesquisa.

Os dados foram organizados em um instrumento criado pelas autoras que aborda a distribuição dos artigos por ano; periódico; metodologia, objetivo, principais resultados e conclusões. As resoluções e as notas técnicas foram distribuídas segundo o ano e principais recomendações.

A análise do conteúdo das publicações incluídas nesta pesquisa foi delimitada à luz do modelo Donabediano de avaliação da qualidade, sendo, portanto, analisadas sob a tríade composta pelas dimensões: Estrutura; Processo; e Resultado. ${ }^{4}$

\section{RESUlTADOS}

Na presente revisão integrativa, analisaram-se 13 artigos e quatro regulamentações, sendo três resoluções e uma nota técnica.

No Gráfico 1, está apresentada a quantidade de artigos por ano. 
GRÁFICO1. Distribuição dos artigos incluídos na revisão por ano.

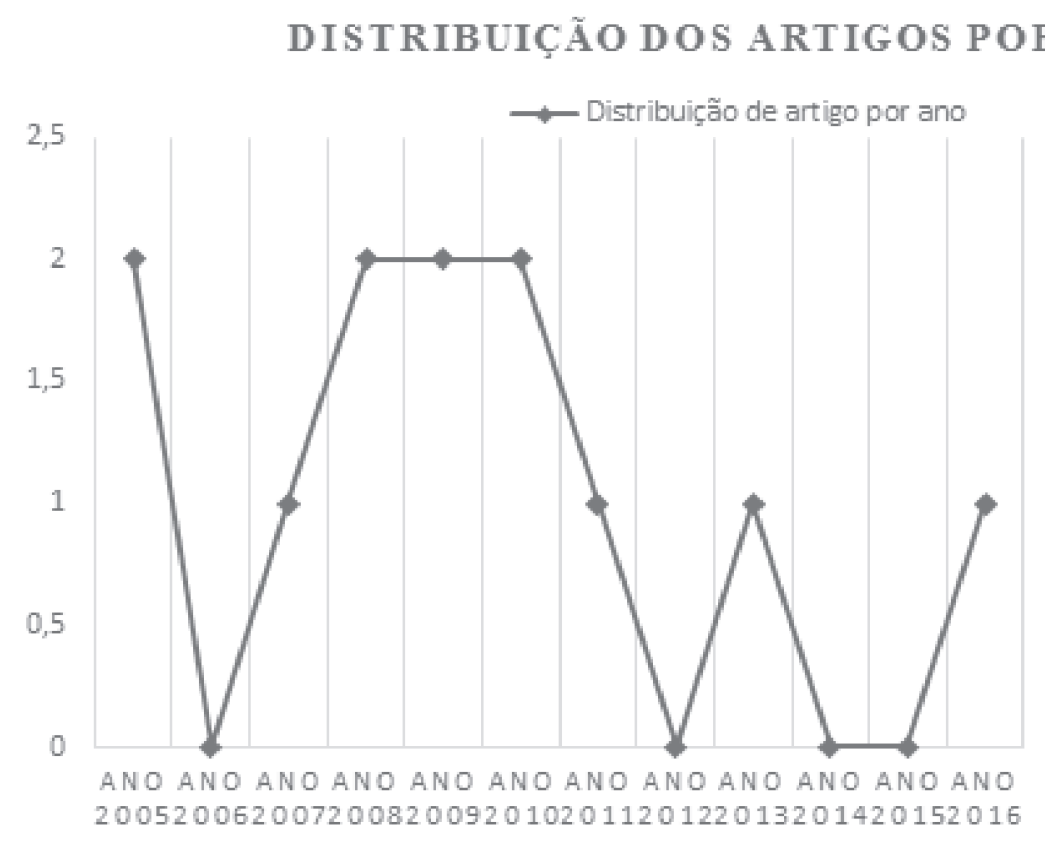

Fonte: Gráfico produzido pelas autoras, Salvador, 2017.

No quadro seguinte encontra-se a distribuição dos artigos publicados pelas revistas.

QUADRO 2. Distribuição da frequência dos artigos incluídos na revisão pelas revistas.

\begin{tabular}{|l|l|}
\hline REVISTA & $(\mathrm{N}) \%$ \\
\hline Revista Acta Paulista de Enfermagem & $(3) 23,07 \%$ \\
\hline Revista do Instituto Adolfo Lutz & $(2) 15,38 \%$ \\
\hline Revista da Escola de Enfermagem da USP & $(2) 15,38 \%$ \\
\hline Boletim Epidemiológico Paulista-BEPA & $(1) 7,69 \%$ \\
\hline Revista Latina Americana de Enfermagem & $(1) 7,69 \%$ \\
\hline Jornal Brasileiro de Nefrologia & $(1) 7,69 \%$ \\
\hline Revista Baiana de Saúde Pública & $(1) 7,69 \%$ \\
\hline Revista de Enfermagem da UERJ & $(1) 7,69 \%$ \\
\hline Revista Eletrônica da Facemed & $(1) 7,69 \%$ \\
\hline
\end{tabular}

Fonte: Quadro produzido pelas autoras, Salvador, 2017. 
No quadro a seguir encontra-se a síntese dos estudos incluídos nesta revisão, suas características e a classificação pelas dimensões de avaliação da qualidade de Avedis Donabedian.

QUADRO 3. Distribuição dos artigos, conforme suas características e sua classificação pelas dimensões de avaliação da qualidade de Avedis Donabedian.

\begin{tabular}{|c|c|c|c|c|}
\hline $\begin{array}{l}\text { Identificação dos artigos: Código/ } \\
\text { Título/Autoria/Ano e Revista }\end{array}$ & Metodologia & Objetivo & $\begin{array}{l}\text { Principais } \\
\text { Resultados e } \\
\text { conclusões }\end{array}$ & Dimensões \\
\hline $\begin{array}{l}\text { A-1 Água de diálise: parâmetros físico- } \\
\text { químicos na avaliação do desempenho } \\
\text { das membranas de osmose reversa } \\
\text { (SIMÕES, M. et al., 2005), Revista do } \\
\text { Instituto Adolfo Lutz. }\end{array}$ & $\begin{array}{l}\text { Estudo de vigilância. } \\
\text { Foram analisadas } 36 \\
\text { amostras de água, } 18 \\
\text { coletadas no cavalete de } \\
\text { entrada (P1) e } 18 \text { após } \\
\text { osmose reversa (P2). }\end{array}$ & $\begin{array}{l}\text { Avaliar o } \\
\text { desempenho das } \\
\text { membranas de } \\
\text { osmose reversa. }\end{array}$ & $\begin{array}{l}100 \% \text { das } \\
\text { amostras de água } \\
\text { apresentaram } \\
\text { conformidade. }\end{array}$ & Estrutura \\
\hline $\begin{array}{l}\text { A2- Correlação entre métodos de } \\
\text { avaliação da qualidade da dose de } \\
\text { diálise (INOUE, K. C. et al., 2009), } \\
\text { Revista Acta Paulista de Enfermagem. }\end{array}$ & $\begin{array}{l}\text { Estudo transversal, } \\
\text { retrospectivo, com } 14 \\
\text { pacientes submetidos a } \\
106 \text { hemodiálises com } \\
\text { a máquina Fresenius } \\
\text { 4008S. }\end{array}$ & $\begin{array}{l}\text { Verificar a } \\
\text { correlação entre o } \\
\text { Kt/V calculado e o } \\
\text { Kt/V-OCM. }\end{array}$ & $\begin{array}{l}\text { Houve diferença } \\
\text { entre o "Kt/V } \\
\text { calculado" e o } \\
\text { "Kt/V máquina". }\end{array}$ & Estrutura \\
\hline $\begin{array}{l}\text { A3- Elaboração de um instrumento da } \\
\text { assistência de enfermagem na unidade } \\
\text { de hemodiálise (OLIVEIRA, S. M. } \\
\text { et al., 2007), Revista Acta Paulista de } \\
\text { Enfermagem. }\end{array}$ & $\begin{array}{l}\text { Estudo longitudinal } \\
\text { prospectivo do tipo } \\
\text { coorte na elaboração } \\
\text { de um instrumento } \\
\text { para a Sistematização } \\
\text { da Assistência de } \\
\text { Enfermagem (SAE). }\end{array}$ & $\begin{array}{l}\text { Construir e testar } \\
\text { um instrumento } \\
\text { para registro da } \\
\text { SAE. }\end{array}$ & $\begin{array}{l}\text { O instrumento } \\
\text { facilita tanto o } \\
\text { registro como a } \\
\text { recuperação de } \\
\text { dados. }\end{array}$ & $\begin{array}{l}\text { Estrutura e } \\
\text { Processo }\end{array}$ \\
\hline $\begin{array}{l}\text { A-4 Projeto de reativação e } \\
\text { implantação do Programa de } \\
\text { Monitoramento da Água Tratada para } \\
\text { Hemodiálise do Estado de São Paulo } \\
\text { (MARCATTO, M. I. S. J. et al., 2008), } \\
\text { Boletim Epidemiológico Paulista. }\end{array}$ & $\begin{array}{l}\text { Estudo de vigilância: } \\
\text { onde foi realizado uma } \\
\text { investigação sanitária } \\
\text { na capital de São } \\
\text { Paulo, adotando como } \\
\text { referência a RDC 154/04 } \\
\text { da ANVISA. }\end{array}$ & $\begin{array}{l}\text { Monitorar a } \\
\text { qualidade da } \\
\text { prestação dos } \\
\text { serviços de diálise. }\end{array}$ & $\begin{array}{l}80 \% \text { das amostras } \\
\text { de água tratada } \\
\text { utilizada em } \\
\text { diálise apresentam } \\
\text { inconformidades. }\end{array}$ & Estrutura \\
\hline $\begin{array}{l}\text { A-5 Metodologia para implantação } \\
\text { de um sistema de gestão da qualidade } \\
\text { em um centro de diálise (LIMA, G. O. } \\
\text { et al., 2009), Revista Acta Paulista de } \\
\text { Enfermagem. }\end{array}$ & $\begin{array}{l}\text { Relato de experiência: } \\
\text { implantar um Sistema de } \\
\text { Gestão da Qualidade em } \\
\text { um Centro de Diálise } \\
\text { em São Luís-MA. }\end{array}$ & $\begin{array}{l}\text { Apresentar a } \\
\text { metodologia } \\
\text { utilizada para } \\
\text { implantar um } \\
\text { Sistema de Gestão } \\
\text { da Qualidade em } \\
\text { um Centro de } \\
\text { Diálise. }\end{array}$ & $\begin{array}{l}\text { Necessidade } \\
\text { de padronizar, } \\
\text { aprovar e validar } \\
\text { documentos; } \\
\text { Estabelecer metas } \\
\text { de acordo com } \\
\text { as medições de } \\
\text { indicadores. }\end{array}$ & $\begin{array}{l}\text { Estrutura e } \\
\text { Processo }\end{array}$ \\
\hline
\end{tabular}




\section{continuação do Quadro 3. Distribuição dos artigos, conforme suas características e sua} classificação pelas dimensões de avaliação da qualidade de Avedis Donabedian.

\begin{tabular}{|c|c|c|c|c|}
\hline $\begin{array}{l}\text { A-6 Indicadores para avaliação do } \\
\text { acesso vascular de usuários em } \\
\text { hemodiálise (NICOLE, A. G. et al., } \\
\text { 2010), Revista Escola de Enfermagem } \\
\text { USP. }\end{array}$ & $\begin{array}{l}\text { Estudo metodológico } \\
\text { para construção } \\
\text { e validação de } \\
\text { indicadores assistenciais } \\
\text { relacionados ao acesso } \\
\text { vascular de usuários de } \\
\text { hemodiálise. }\end{array}$ & $\begin{array}{l}\text { Construir } \\
\text { indicadores para } \\
\text { avaliar a qualidade } \\
\text { das práticas } \\
\text { assistenciais } \\
\text { relacionadas ao } \\
\text { acesso vascular. }\end{array}$ & $\begin{array}{l}\text { Foram construídos } \\
\text { quatro indicadores } \\
\text { acerca de cateter } \\
\text { temporário e } \\
\text { fístula. Todos } \\
\text { foram validados. }\end{array}$ & Estrutura \\
\hline $\begin{array}{l}\text { A-7 A importância de programas de } \\
\text { monitoramento da qualidade da água } \\
\text { para diálise na segurança dos pacientes } \\
\text { (BUZZO, M. L. et al., 2010), Revista do } \\
\text { Instituto Adolfo Lutz. }\end{array}$ & $\begin{array}{l}\text { Estudo epidemiológico. } \\
\text { O monitoramento foi } \\
\text { delineado para avaliar } \\
\text { os serviços de diálise } \\
\text { quanto à qualidade } \\
\text { da água utilizada no } \\
\text { tratamento. }\end{array}$ & $\begin{array}{l}\text { Descrever a } \\
\text { condição das } \\
\text { clínicas de diálise } \\
\text { do Estado no que se } \\
\text { refere à qualidade } \\
\text { da água. }\end{array}$ & $\begin{array}{l}13 \text { amostras de } \\
2008 \text { e cinco } \\
\text { amostras de } 2009, \\
\text { coletadas no } \\
\text { ponto contíguo } \\
\text { à máquina, } \\
\text { indicaram falha na } \\
\text { coleta. }\end{array}$ & Estrutura \\
\hline $\begin{array}{l}\text { A-8 Avaliação da conformidade da } \\
\text { prática assistencial de manutenção do } \\
\text { cateter temporário duplo lúmen para } \\
\text { hemodiálise (ROSETTI, K. A. G. et } \\
\text { al. 2014), Revista latina Americana de } \\
\text { Enfermagem. }\end{array}$ & $\begin{array}{l}\text { Estudo de abordagem } \\
\text { quantitativa, } \\
\text { exploratório-descritivo, } \\
\text { observacional, } \\
\text { realizado na unidade de } \\
\text { hemodiálise. }\end{array}$ & $\begin{array}{l}\text { Avaliar a } \\
\text { conformidade da } \\
\text { prática assistencial } \\
\text { de manutenção do } \\
\text { cateter temporário } \\
\text { duplo lúmen. }\end{array}$ & $\begin{array}{l}99,4 \% \text { em } \\
\text { conformidade do } \\
\text { uso de máscaras } \\
\text { na conexão e } 100 \% \\
\text { na desconexão. } \\
35,6 \% \text { de adesão } \\
\text { à prática de } \\
\text { higienização das } \\
\text { mãos. }\end{array}$ & $\begin{array}{l}\text { Estrutura e } \\
\text { Processo }\end{array}$ \\
\hline $\begin{array}{l}\text { A-9 Avaliação do Desempenho } \\
\text { Operacional de Dialisadores de Fibra } \\
\text { Oca de Polietersulfona em Condições } \\
\text { de usos Múltiplos (CASTRO, M. C. } \\
\text { M. et al., 2008), Jornal Brasileiro de } \\
\text { Nefrologia. }\end{array}$ & $\begin{array}{l}\text { A membrana do } \\
\text { filtro de hemodiálise } \\
\text { foi modificada de } \\
\text { polisulfona para } \\
\text { polietersulfona. }\end{array}$ & $\begin{array}{l}\text { Avaliar o } \\
\text { desempenho } \\
\text { de dialisadores } \\
\text { de fibra oca de } \\
\text { polietersulfona na } \\
\text { condição de usos } \\
\text { múltiplos. }\end{array}$ & $\begin{array}{l}\text { Os dialisadores } \\
\text { de polietersulfona } \\
\text { testados neste } \\
\text { estudo mostraram } \\
\text { um bom } \\
\text { desempenho. }\end{array}$ & $\begin{array}{l}\text { Estrutura e } \\
\text { Resultado }\end{array}$ \\
\hline $\begin{array}{l}\text { A-10 A legislação sanitária e a } \\
\text { qualidade da água nas clínicas de } \\
\text { hemodiálise de Salvador (OLIVEIRA, } \\
\text { I. C. P.), 2005. Revista Baiana de Saúde } \\
\text { Pública. }\end{array}$ & $\begin{array}{l}\text { Estudo de Vigilância. } \\
\text { Foi desenvolvido um } \\
\text { estudo em seis clínicas } \\
\text { de hemodiálise. }\end{array}$ & $\begin{array}{l}\text { Avaliar a qualidade } \\
\text { da água utilizada } \\
\text { na HD, com base } \\
\text { na Portaria MS } \\
n^{\circ} .82 / 00 \text { e da } \\
\text { Resolução de } \\
\text { Diretoria Colegiada } \\
\left(\text { RDC) } n^{\circ} .154 / 04 .\right.\end{array}$ & $\begin{array}{l}\text { O teste de } \\
\text { qualidade da } \\
\text { água é realizado } \\
\text { diariamente em } \\
\text { todas as clínicas, } \\
\text { e o controle } \\
\text { microbiológico } \\
\text { é verificado } \\
\text { mensalmente em } \\
\text { cinco clínicas. }\end{array}$ & Estrutura \\
\hline
\end{tabular}


continuação do Quadro 3. Distribuição dos artigos, conforme suas características e sua classificação pelas dimensões de avaliação da qualidade de Avedis Donabedian.

\begin{tabular}{|c|c|c|c|c|}
\hline $\begin{array}{l}\text { A-11 Adverse events in hemodialysis: } \\
\text { reports of nursing professionals } \\
\text { (SOUZA, M. R. G. et al., 2013) Revista } \\
\text { da Escola de Enfermagem da USP. }\end{array}$ & $\begin{array}{l}\text { Estudo transversal } \\
\text { e quantitativo que } \\
\text { analisou o conhecimento } \\
\text { dos profissionais de } \\
\text { enfermagem sobre } \\
\text { Eventos Adversos (EA). }\end{array}$ & $\begin{array}{l}\text { Analisar o } \\
\text { conhecimento dos } \\
\text { trabalhadores de } \\
\text { enfermagem em } \\
\text { relação à EA. }\end{array}$ & $\begin{array}{l}12 \text { dos } 25 \\
\text { trabalhadores não } \\
\text { foram capazes de } \\
\text { definir eventos } \\
\text { adversos. }\end{array}$ & $\begin{array}{l}\text { Estrutura e } \\
\text { Resultado }\end{array}$ \\
\hline $\begin{array}{l}\text { cA-12 Avaliação da técnica de curativo } \\
\text { em cliente com acesso venoso para } \\
\text { hemodiálise (NASCIMENTO, V. C. } \\
\text { P., 2009) Revista de Enfermagem } \\
\text { da Universidade Estadual do Rio de } \\
\text { Janeiro. }\end{array}$ & $\begin{array}{l}\text { Estudo descritivo, com } \\
\text { abordagem quantitativa, } \\
\text { foi realizado numa } \\
\text { clínica de nefrologia em } \\
\text { Aracaju/SE. }\end{array}$ & $\begin{array}{l}\text { Verificar o } \\
\text { desempenho } \\
\text { da equipe de } \\
\text { enfermagem na } \\
\text { realização do } \\
\text { curativo de clientes } \\
\text { em uso de cateter } \\
\text { venoso para } \\
\text { hemodiálise. }\end{array}$ & $\begin{array}{l}\text { Os Equipamentos } \\
\text { de Proteção } \\
\text { Individual (EPI’s) } \\
\text { mais utilizados } \\
\text { foram: jaleco e } \\
\text { luvas estéreis em } \\
30 \text { (100\%) dos } \\
\text { procedimentos, } \\
\text { máscara em } 29 \\
\text { (96,6\%). }\end{array}$ & $\begin{array}{l}\text { Estrutura e } \\
\text { Processo }\end{array}$ \\
\hline $\begin{array}{l}\text { A-13 Uso do lúdico em hemodiálise: } \\
\text { buscando novas perspectivas na } \\
\text { qualidade de atendimento ao paciente } \\
\text { no centro de diálise (CAVALCANTE } \\
\text { F. A. C. et al., 2011) Revista Eletrônica } \\
\text { da Facemed. }\end{array}$ & $\begin{array}{l}\text { Estudo de caráter } \\
\text { exploratório com } \\
\text { abordagem qualitativa. } \\
\text { Foi realizada atividade } \\
\text { lúdica com pacientes que } \\
\text { participam do programa } \\
\text { de hemodiálise no } \\
\text { Centro de Diálise de } \\
\text { Cacoal (CDC). }\end{array}$ & $\begin{array}{l}\text { Desenvolver } \\
\text { atividades lúdicas, } \\
\text { jogos, pinturas e } \\
\text { dinâmicas para } \\
\text { os pacientes } \\
\text { hemodialisados. }\end{array}$ & $\begin{array}{l}\text { O resultado foram } \\
\text { momentos de } \\
\text { descontração, } \\
\text { alegria e interação. } \\
\text { Apenas um } \\
\text { paciente teve medo } \\
\text { para realizar as } \\
\text { atividades. }\end{array}$ & $\begin{array}{l}\text { Processo e } \\
\text { Resultado }\end{array}$ \\
\hline
\end{tabular}

Fonte: Quadro produzido pelas autoras, Salvador, 2017.

No gráfico a seguir apresenta-se a frequência da distribuição dos artigos pelas dimensões de avaliação da qualidade, segundo Avedis Donabedian.

GRÁFICO 2. Distribuição dos artigos, segundo modelo de avaliação de qualidade de Donabedian.

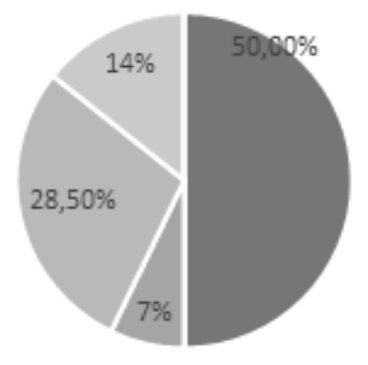

$$
\text { - Estrutura | Processo + resul tado "Estrutura + Processo "Estruturatresultado }
$$

Fonte: Gráfico produzido pelas autoras, Salvador, 2017. 
No quadro a seguir encontra-se a apresentação das regulamentações que abordam aspectos relevantes para a qualidade em diálise, bem como, o ano de publicação, principais recomendações, e sua classificação conforme dimensão de qualidade, segundo Avedis Donabedian.

Quadro 4. Síntese das regulamentações, de acordo com o ano, recomendações, e classificação da dimensão da qualidade, conforme a tríade Donabediana.

\begin{tabular}{|c|c|c|c|}
\hline Regulamentações & Ano & Recomendações & Dimensão \\
\hline $\begin{array}{l}\text { Portaria No. } 2.042 \text {, de } \\
11 \text { de outubro } 1996- \\
\text { SUBSTITUÍDA pela } \\
\text { Resolução - RDC No. } \\
154 \text {, de } 15 \text { de junho de } \\
2004 .\end{array}$ & 1996 & $\begin{array}{l}\text { Utilização de um dialisador e das linhas arteriais e venosas por até } 6 \text { vezes } \\
\text { para o mesmo paciente, empregando técnica de reuso. } \\
\text { Pacientes portadores de HIV: não será permitido o reuso dos dialisadores e } \\
\text { linhas arteriais e venosas. } \\
\text { Estabelece recursos materiais e recursos humanos. } \\
\text { Estabelece o padrão de qualidade da água tratada utilizada na preparação de } \\
\text { solução para diálise. Contagem de bactérias heterotróficas. }\end{array}$ & Estrutura \\
\hline $\begin{array}{l}\text { Resolução - RDC No. } \\
\text { 154, de } 15 \text { de junho de } \\
2004 \text { - SUBSTITUÍDA } \\
\text { pela Resolução - RDC } \\
\text { No. } 11 \text {, de } 13 \text { de março } \\
\text { de } 2014 .\end{array}$ & 2004 & $\begin{array}{l}\text { Substitui de } 6 \text { para } 12 \text { a quantidade de vezes de utilização de dialisadores } \\
\text { e as linhas arteriais e para o mesmo paciente, quando empregado o } \\
\text { reprocessamento manual, ou até } 20 \text { (vinte) vezes quando empregado } \\
\text { reprocessamento automático. } \\
\text { Altera o nível de ação relacionado à contagem de bactérias heterotróficas de } \\
100 \text { para } 50 \text { ufc/ml. } \\
\text { Define a equipe mínima do quadro de diálise ( } 02 \text { enfermeiros; } 02 \text { médicos; } 01 \\
\text { assistente social; } 01 \text { psicólogo; } 01 \text { nutricionista; } 01 \text { auxiliar de enfermagem; } 01 \\
\text { técnico de enfermagem; } 01 \text { funcionário para o serviço de limpeza. } \\
\text { Define o quantitativo de pacientes para cada profissional: } 01 \text { enfermeiro para } \\
\text { cada } 35 ; 01 \text { enfermeiro para cada } 35 ; 01 \text { técnico ou auxiliar para cada } 04 \\
\text { pacientes. } \\
\text { Define infraestrutura para unidade de diálise. }\end{array}$ & Estrutura \\
\hline $\begin{array}{l}\text { Nota Técnica No. } \\
\text { 006/2009 -GGTES/ } \\
\text { ANVISA - EM VIGOR. }\end{array}$ & 2009 & $\begin{array}{l}\text { Determina os parâmetros para o uso de diálise em UTI's. } \\
\text { Determina que a unidade deve possuir osmose reversa portátil. } \\
\text { Proíbe o reuso de agulhas, dialisadores, linhas, isoladores de pressão e demais } \\
\text { materiais descartáveis bem como sobras de medicamentos, concentrado } \\
\text { polieletrolítico (CPHD) e dialisadores. }\end{array}$ & Estrutura \\
\hline $\begin{array}{l}\text { Resolução - RDC No. } 11 \text {, } \\
\text { de } 13 \text { de março de } 2014 \text { - } \\
\text { EM VIGOR. }\end{array}$ & 2014 & $\begin{array}{l}\text { Determina que o serviço de diálise deve constituir um Núcleo de Segurança } \\
\text { do Paciente. } \\
\text { Acrescenta a proibição de reuso de dialisadores de paciente com sorologia } \\
\text { positiva para hepatite B, hepatite C (tratados ou não). } \\
\text { Fica proibido o reuso de linhas arteriais e venosas utilizadas em todos os } \\
\text { procedimentos hemodialíticos. } \\
\text { Fica determinado que os dialisadores podem ser utilizados para o mesmo } \\
\text { paciente no máximo } 20 \text { (vinte) vezes, após ser submetido ao processamento } \\
\text { automático em vez de } 12 \text { em processamento manual. }\end{array}$ & Estrutura \\
\hline
\end{tabular}

Fonte: Quadro produzido pelas autoras, Salvador, 2017. 


\section{Discussão}

No que tange ao Gráfico 1, notam-se duas publicações, em 2009. Tal acontecimento pode ser atribuído à implementação da Nota Técnica do Ministério da Saúde No. 006/2009-GGTES/ANVISA, ${ }^{9}$ que estabeleceu parâmetros para a execução de procedimentos dialíticos em ambiente hospitalar, em unidades de terapia intensiva, nas quais ocorre diálise, logo após abrangida pela RDC/Anvisa n. 154, de 15 de junho de $2014 .^{10}$

Com base no Quadro 2, evidencia-se que há concentração de publicações científicas na Revista Acta Paulista de Enfermagem, com três publicações (A2, A3, A5), seguida pela Revista do Instituto Adolfo Lutz, com duas publicações (A1 e A7), as demais revistas apresentaram apenas uma publicação. ${ }^{11,12,13,14,15}$

Segundo o modelo de Donabedian, a tríade "Estrutura, processo e resultado" abrange todas as atividades desenvolvidas entre profissionais de saúde e os pacientes em um ambiente, nessa relação cada componente pode ser avaliado separadamente, mas para alcançar um resultado significativo, as três dimensões devem ser avaliadas juntas de forma sistêmica. ${ }^{16}$

Além da tríade, Donabedian estabelece ainda alguns critérios para avaliar o processo de qualidade, sendo eles: 1) eficácia: a capacidade de cuidar, no seu melhor, para melhorar a saúde; 2) efetividade: o grau em que as melhorias de saúde atingíveis são realizadas; 3) eficiência: a capacidade de obter a maior melhoria da saúde ao menor custo; 4) otimismo: o equilíbrio mais vantajoso de custos e benefícios; 5) aceitabilidade: conformidade com as preferências dos pa- cientes em relação à acessibilidade, a relação paciente-profissional, as comodidades, os efeitos dos cuidados e o custo dos cuidados; 6) legitimidade: conformidade com as preferências sociais em relação a todos os itens citados; e 7) equidade: igualdade na distribuição dos cuidados e dos seus efeitos sobre a saúde. ${ }^{3}$

Nesse entendimento de qualidade, com relação aos estudos incluídos nesta revisão, foi verificado que 93\% dos artigos (A1); (A2); (A3); (A4); (A5); (A6); (A7); (A8); (A9); (A10); (A11); (A12) avaliam qualidade relacionada à dimensão estrutura. Esse achado deve-se ao fato de a estrutura ser mais fácil de ser medida e observada, podendo ser entendida também como uma das causas dos problemas nos processos e nos resultados. ${ }^{14,11,12,17,13,18,15,19,20,12,22,23}$

Os artigos (A1), (A4), (A7) fazem parte da dimensão estrutura da tríade e analisam os parâmetros físico-químicos da água, como subsídio importante de avaliação do desempenho das membranas de osmose reversa responsáveis pelo tratamento da água de diálise. Os pacientes submetidos ao tratamento regular de hemodiálise estão expostos semanalmente a uma grande quantidade de água de diálise por meio da circulação extracorpórea, em torno de 150 litros/turno. Até a década de 70, acreditava-se que a água potável servia para o tratamento dialítico, porém com o aumento de usuários no tratamento, pôde-se perceber que os contaminantes da água potável poderiam levar a efeitos adversos, podendo ser letais à vida do paciente, haja vista a tragédia ocorrida com pacientes em tratamento de hemodiálise em Pernambuco, no ano de 1996. Em uma sessão de diálise, o uso de água pura para forma- 
ção do composto dialisante é pré-requisito para o funcionamento da instituição, naturalmente não se tem água pura, sendo necessária a realização de tratamento para a obtenção da mesma. ${ }^{14,17,15}$

Após esse evento, surge a necessidade da portaria 2.042, de 1996, sendo a primeira portaria que estabeleceu critérios de verificação da potabilidade da água de abastecimento, e determinou a responsabilidade do controle da mesma. Em seguida, publicou-se a RDC 154, de 2004, que estabeleceu que a água de abastecimento dos serviços de diálise proveniente da rede pública, de poços artesianos ou de outros mananciais, deve ter o seu padrão de potabilidade em conformidade com o disposto na Portaria GM/MS no. 518, de 25 de março de 2004, que estabelece padrões de potabilidade para o consumo humano. Em 2009, a Nota técnica №. 006 estabeleceu o uso de osmose reversa para o tratamento de diálise em ambientes intra-hospitalar fora de serviço de diálise. ${ }^{9,10,24}$

A Osmose reversa é um sistema de tratamento de água geralmente composto por: filtro de areia, abrandador, filtro de carvão ativado. ${ }^{25}$ Esse processo é importante para o tratamento de diálise e surge da necessidade de se evitar contaminações pela água, evitando assim eventos adversos relacionados ao processo do tratamento da água. ${ }^{14,17,15}$

A portaria 154 de 2014, vigente, estabelece todos os parâmetros para a avaliação da qualidade da água, porém não determina qualquer especificidade de formação para a atuação nesse campo e diante da complexidade e da importância do trabalho realizado pelos técnicos de tratamento da água, seria interessante que a sua capacitação fosse também de órgão responsável pelo setor saúde e não oferecido unicamente por empresa que efetua a venda do equipamento, dando possibilidade de legitimidade e de redução de possíveis erros, com a padronização dos processos e melhoria de resultados. ${ }^{21}$

Um número expressivo de artigos abordou a questão da água para diálise, tal fato pode ser atribuído aos problemas imediatos que estão relacionados à não conformidades nesse componente do tratamento. Dessa forma, medir a qualidade da água, bem como, a obtenção da água com parâmetros ideais, tornou-se um aspecto de grande relevância na garantia de uma assistência eficaz, eficiente e efetiva aos pacientes dialíticos.

O Artigo (A2) foi classificado no componente estrutura, o referido estudo fez comparação entre o uso do Kt/V calculado pela fórmula de Daugirdas e os resultados mensurados pelo Monitor (OCM) da máquina de diálise. A quantidade de ureia removida é o padrão chave para a evolução da eficiência de uma seção de hemodiálise. Geralmente, o cálculo dessa remoção se baseia no Kt/V que dá uma mensuração na distribuição de ureia no corpo. Essa tecnologia otimiza o atendimento, pois prevê o Kt/V em tempo real, podendo quantificar a dose de diálise necessária para o paciente no momento, otimizando o atendimento e a segurança do paciente. A garantia de estrutura ideal para o cálculo fidedigno do Kt/V é premissa para uma sessão de hemodiálise. Desse modo, o uso de tecnologias que permitam o cálculo de maneira eficiente e eficaz proporciona melhorar a qualidade da assistência e garantir processos mais adequados visando o melhor resultado aos pacientes em tratamento. ${ }^{11}$ 
Dados como esse são importantes e devem estar em formulários elaborados, como o do artigo (A3), que formaliza as informações de diálise do paciente por meio da Sistematização da Assistência de Enfermagem, que é dividido em cinco fases: coleta de dados, diagnóstico, plano de ação, evolução e implementação. A partir desses dados, é possível verificar o processo do cuidado que foi planejado diariamente para cada paciente que realiza a hemodiálise, bem como, avaliar a sua evolução ao longo do tratamento. ${ }^{12}$

A sistematização da assistência proporciona uma avaliação global dos cuidados aos pacientes, que podem ser utilizados como relatórios para propor melhorias e organizar uma gestão de qualidade. O artigo (A5), ao trabalhar com a gestão da qualidade, visa atender às demandas dos clientes e alcançar a satisfação dos mesmos. Utilizando o princípio da gestão da qualidade pela instituição para planejar, identificar e controlar processos, promovendo a organização do ambiente de trabalho, a capacitação dos recursos humanos, análise crítica e satisfação dos clientes, promovendo melhoria contínua. ${ }^{13}$

A qualidade da assistência à saúde pode ser definida como obtenção de maiores benefícios em detrimentos de menores riscos para o paciente, com condições materiais e funcionais. ${ }^{26}$ Assim, o resultado deve ser sempre analisado se é compatível com a organização e com o planejamento. O artigo (A6) trata da criação de indicadores de qualidade relacionado ao desempenho e à manutenção do Cateter Duplo Lúmen (CDL) para hemodiálise, e monitoramento e complicações das fístulas arteriovenosas. Sabendo que uma boa via de acesso é fundamental para a realização de hemodiálise, esses indicadores tornam-se essenciais para proporcionar a qualidade da assistência no tratamento dialítico. ${ }^{18}$ A construção e a validação de indicadores relacionados a acessos venosos podem ser o primeiro passo para proporcionar estrutura para garantir a sua permanência, viabilidade, prevenção de infecções e complicações, sendo, portanto, fundamental para a realização da sessão de diálise sem intercorrências.

Alguns indicadores como a Taxa de Mortalidade, a taxa de Saída por Transplante, a taxa de Soroconversão para Hepatite C, a taxa de Internação dos Pacientes em diálise e a taxa de Pacientes em Uso de Cateter Venoso Central Temporário, são indicadores de resultado, e já são estabelecidos na portaria 154 de $2004 .^{27}$

O Monitoramento por meio de indicadores pode ser definido como uma atividade sistemática, para a possível avaliação e implementação de medidas. Os principais indicadores de segurança do paciente são: Indicadores ou eventos sentinela: aqueles em que só um caso é suficiente para indicar o problema; Indicadores de resultado: referem-se à presença de complicações; Indicadores de boas práticas: trata-se de um enfoque alternativo ou complementar ancorado em medir a presença de aspectos estruturais e de processo. Esses indicadores quantificam os resultados nos pacientes, quando se tem um evento não característico da unidade ou que foge à realidade. ${ }^{28}$

Ao se analisar as vantagens do uso dos indicadores de qualidade do processo e do resultado na realização de práticas seguras, outros autores (A8) e (A12) vêm detalhando a avaliação de indicadores de processo: manutenção do CDL por meio da realização 
do curativo com técnica correta mediante a realização da higienização das mãos, da técnica estéril, do uso de EPIs, da inspeção e da palpação de orifício, do tempo de trocas corretas e do preenchimento de vias corretas. Esses indicadores são ferramentas importantes para a avaliação do processo, pois subsidiam a prática gerencial e assistencial, dirigindo a atenção dos profissionais para a importância da conformidade na execução dos processos, sustentando melhorias contínuas no atendimento prestado. ${ }^{19,23}$

$\mathrm{Na}$ avaliação da qualidade no que tange a recursos materiais, o artigo (A9) avalia o uso de diferentes membranas do dialisado. Nesse estudo, a membrana do filtro de hemodiálise foi modificada de polisulfona para polietersulfona, essa membrana é utilizada na diálise para filtrar o sangue do paciente, ela é como um rim artificial, por onde ocorre a filtração, é nesse processo que são retidas as escórias do paciente. A qualidade da membrana do dialisado interfere diretamente na realização de uma boa diálise, influenciando o risco de infecção por rupturas, por isso os recursos materiais de boa fabricação compõem a categoria de estrutura e interferem diretamente na eficácia de uma boa diálise. ${ }^{20} \mathrm{~A}$ partir deste estudo, visualiza-se a importância de recursos materiais adequados para a garantia da qualidade da sessão de diálise, salienta-se que a portaria 154 de 2004 estabelece o uso apenas de membrana biocompatíveis. ${ }^{27}$

O artigo (A11) avalia a capacidade tecnicocientífica do profissional enfermeiro ao tentar mensurar o conhecimento dos mesmos sobre a identificação de eventos adversos em hemodiálise; os tipos de condutas do cuidar da enfermagem em relação a esses eventos; a ocorrência desse tipo de evento na instituição estudada; os seus fatores causais; as medidas tomadas; e as quais sugestões dos profissionais em relação aos eventos. A verificação do conhecimento dos profissionais poderá gerar condutas eficazes como: planos de contingência e educação permanente, otimizando o cuidado. ${ }^{22} \mathrm{~A}$ equipe de enfermagem precisa conhecer $\mathrm{o}$ processo de diálise como um todo e quão frágil ele pode ser, para que os mesmos identifiquem potenciais falhas e evitem que estas ocorram. Assim, o conhecimento será um forte aliado do cuidado seguro e com qualidade. À gerência de enfermagem fica a responsabilidade de estabelecer protocolos para a realização de procedimentos da diálise, esses protocolos não devem mecanizar o processo e sim auxiliar a fim de evitar os eventos adversos. ${ }^{29}$ Por isso, a RDC 154 estabelece que todo o serviço de diálise deve implantar e implementar um Programa de Controle e Prevenção de Infecção e de Eventos Adversos (PCPIEA). ${ }^{27}$

O Estudo (A13) trabalha com a implantação de programas lúdicos como jogos, pinturas e dinâmicas para os pacientes hemodialisados. Nesse trabalho, as avaliações dos resultados são demonstradas por meio de escalas, cada resultado está diretamente ligado ao processo realizado pelos pacientes que podem ser uma pintura ou um jogo. Assim, é vista a aceitabilidade dos pacientes e a legitimidade dos resultados de acordo com as escalas quantitativas e qualitativas. ${ }^{30}$ O cliente portador de IRC, em Programa de Hemodiálise, padece de uma patologia que, além de diminuir-lhe grande parte de sua capacidade física, impõe-lhe uma rotina de tratamento tão intensa que modifica 
seu modo de viver e influencia desfavoravelmente a sua qualidade de vida. A utilização do lúdico pelas enfermeiras nas relações inter-humanas voltadas ao cuidado para o bem-estar das pessoas em hemodiálise pode proporcionar outras formas de ver a vida e a doença, de maneira mais descontraída e mais feliz. ${ }^{31}$ Tais aspectos encontrados revelam a importância de abordar-se esse tema relacionando-o com a qualidade da assistência, e por conseguinte com mais qualidade de vida para os pacientes.

As RDCs são normas criadas por representações diversas e tomadas em grupo com o aproveitamento de experiências diferenciadas. Já a Nota Técnica é um documento elaborado por técnicos especializados em determinado assunto e difere do Parecer pela análise completa de todo o contexto, devendo conter histórico e fundamento legal, ancorados em informações relevantes. ${ }^{32} \mathrm{O}$ reconhecimento do risco potencial que representava a ausência de um tratamento específico para a água utilizada em diálise levou à criação, no Brasil, em outubro de 1996, da Portaria 2.042 do Ministério da Saúde, para tratar, especificamente, da qualidade da água para hemodiálise substituída pela Portaria $n^{\circ}$. 82, de janeiro de 2000, pela RDC no. 154, de 15 de junho de 2004. Atualmente, está em vigor a RDC No. 11, de 13 de março de 2014, que dispõe sobre os requisitos de boas práticas de funcionamento para os serviços de diálise e dá outras providências. ${ }^{24,33,27,34}$

As portarias consideram a necessidade de redefinir os critérios mínimos para o funcionamento e a avaliação dos serviços públicos e privados que realizam diálise em pacientes ambulatoriais, portadores de insu- ficiência renal crônica, bem como, os mecanismos de sua monitoração, garantiram em sua evolução histórica mudanças que ajustam valores de padrão de qualidade da água, e outros recursos mínimos necessários para a realização de diálise.

Essas normas são elaboradas visando à avaliação conforme critérios previamente definidos, possibilitando o ajuste de condutas individuais e a gestão dos processos assistenciais e gerenciais, devendo possuir vigência, ou seja, devendo apresentar-se válida. A cada nova publicação há invalidação das anteriores, pois, no decorrer dos anos, avanços no conhecimento e tecnologias em diálise ancoradas em evidências científica, possibilitaram uma melhoria na qualidade do atendimento, na medida em que houve novas técnicas científicas e surgimento de novas máquinas de diálise com tecnologias mais eficazes e efetivas.

\section{CONSIDERAÇões FINAIS}

Ao conhecer e classificar as produções científicas que abordam a qualidade em serviço de diálise, no Brasil, pôde-se perceber que $50 \%$ comtemplaram somente o componente estrutura e outras a abordaram em parceria com outra dimensão. Assim, pôde ser observado que há uma preocupação com relação à adequação dos padrões estruturais estabelecidos pelas regulamentações. O que de fato é importante, mas é plausível fazer a reflexão que apenas a estrutura não possibilita a garantia de se ter uma qualidade na assistência, visto que Donabedian preconiza uma assistência ancorada na tríade de forma sistêmica. Faz-se necessário compreender que a estrutura adequada é um fator 
subsidiador da qualidade, mas é por meio da relação estabelecida no processo que os resultados são delimitados, referenciando a intersecção das três dimensões.

Diante da revisão integrativa realizada, verificamos que muitos dos artigos abordaram as regularidades e irregularidades nos padrões limítrofes das portarias. Os processos de trabalho foram avaliados por alguns estudos por meio de indicadores de qualidade e de observação da técnica, mostrando a importância de manter uma conformidade na execução dos procedimentos, garantindo melhorias contínuas e resultados positivos. É válido salientar que cabe também à gestão a promoção de aprimoramentos, vigilância contínua, para que os profissionais realizem as ações com segurança, eficiência e efetividade, garantindo bons resultados aos pacientes.

Alguns textos trabalharam a observação de processos de trabalho para a realização do tratamento da água, para a realização de curativo, manejo adequado de CDL, além de implantação de avaliação de satisfação de usuário. Pesquisas assim são importantes, pois possibilitam avaliar os resultados de cada processo para o usuário do serviço. Outros artigos analisaram os eventos adversos, o uso de atividade lúdica em paciente, e a troca de membranas de diálise, todos esses estudos avaliam os efeitos de um atendimento sobre o paciente incluindo alterações ao estado de saúde, comportamento ou conhecimento, bem como, a satisfação do paciente e da saúde relacionados com a qualidade de vida.

Os resultados são muitas vezes vistos como os mais importantes indicadores de qualidade, porque a repercussão do cuidado no estado de saúde do paciente é o principal objetivo dos cuidados assistidos, porém se evidencia que a conjunção da tríade é uma forma de garantir uma qualidade mais efetiva e sistêmica nos serviços de saúde, incluindo os de diálise.

Dessa forma, esta revisão elucida como lacuna científica a falta de estudos que contemplem concomitantemente a estrutura necessária, a adequação dos processos e a avaliação por meio dos resultados para os pacientes em tratamento de diálise, sendo assim, evidencia essa lacuna como oportunidades de novos estudos em diálise no Brasil.

\section{REFERÊNCIAS}

1- Pinheiro R., Lima JCF, Pereira IB, Fonseca AF, Martins AS, Mota A. Dicionário da Educação Profissional em Saúde, 2. ed. Rio de Janeiro: Fundação Oswaldo Cruz. Escola Politécnica de Saúde Joaquim Venâncio, 2009. Disponível em: http://www.epsjv.fiocruz.br/dicionario/Dicionario2.pdf.

2- Martins MMFPS, Gonçalves MNC, Ribeiro OMPL, Tronchin, DMR. Quality of nursing care: instrument development and validation. Rev Bras Enferm (on-line) 2016 [acesso em: 06 jun. 2016]; 69 (5): 864-70. Disponível em: http://www.scielo.br/scielo.php?script=sci_arttext\&pid=S 003471672016000500920\&lng=pt\&tlng=pt.

3- Donabedian A. The seven pillars of quality. Archives of Pathology \& Laboratory Medicine, Chicago (on-line) 1990 [acesso em: 21 jun. 2016]; 114 (11): 1115-8. Disponível em: https:// scielosp.org/scieloOrg/php/reflinks.php?refpid=S14138123200800090002700021\&lng=pt\&pid $=$ S1413-81232008000900027. 
4- Donabedian A. The definition of quality and approaches to its assessment: explorations in quality assessment and monitoring. Health Administration Press, Chicago (US), 1988; 260 (12): 743-8.

5- Portela MC. Avaliação da qualidade em saúde. In: Rozenfel, DS (Org.). Fundamentos da Vigilância Sanitária [on-line]. Rio de Janeiro: Editora FIOCRUZ, 2000, p. 259-269 [acesso em: 5 jun. 2016]. Disponível em: http://books.scielo.org/id/d63fk/pdf/rozenfeld-9788575413258-15.pdf.

6- Andrade LM, Martins EC, Caetano JA, Soares E., Beserra, EP. Atendimento humanizado nos serviços de emergência hospitalar na percepção do acompanhante. Rev. Eletr. Enf. [online] 2009 [acesso em: 23 jun. 2016]; 11 (1): 151-7. Disponível em: https://www.fen.ufg.br/revista/ v11/n1/pdf/v11n1a19.pdf

7- Câmara HF. A "tragédia da hemodiálise" 12 anos depois: poderia ela ser evitada? [Tese]. Recife (PE): Fundação Oswaldo Cruz, 2011. Disponível em: http://www.cpqam.fiocruz.br/ bibpdf/2011camaraneto-hf.pdf.

8- Mendes KDS, Silveira RCCP, Galvão CM. Revisão integrativa: método de pesquisa para a incorporação de evidências na saúde e na enfermagem. Texto Contexto Enferm, Florianópolis 2008 [acesso em: 8 jun. 2016]; 17 (4): 758-64. Disponível em: http://www.scielo.br/pdf/tce/ v17n4/18.pdf.

9- Brasil. Nota técnica No. 006/2009-GGTES/ANVISA. Estabelece parâmetros para execução de procedimentos dialíticos em ambiente hospitalar fora dos serviços de diálise. Brasília, 21 de dezembro de 2009 [acesso em: 6 jun. 2016]. Disponível em: https://sbn.org.br/app/uploads/NotaTecnica006-2009-GGTES-ANVISA1.pdf.

10- Brasil. Portaria no ${ }^{\circ}$ 518, de março de 2004, Gabinete do Ministro - Ministério Da Saúde, Diário Oficial da União n⿳0.59 de 26/03/2004, Seção 1, p. 266-70.

11- Inoue KC, Kuroda CN, Nakayama KG, Delliagnolo CM, Matsuda LM. Correlação entre métodos de avaliação da qualidade da dose de diálise. Acta Paul. Enferm. [on-line], 2009 [acesso em: 4 jun. 2016]; 22 (spe1): 494-6. Disponível em: http://www.scielo.br/pdf/ape/v22nspe1/07.pdf.

12- Oliveira SM, Ribeiro RCHM, Ribeiro DF, Lima LCEQ, Pinto MH, Poletti NAA. Elaboração de um instrumento da assistência de enfermagem na unidade de hemodiálise. Acta Paul Enferm 2008 [acesso em: 25 jun. 2016]; 21 (Número Especial): 169-73. Disponível em: http://www.scielo.br/pdf/ape/v21nspe/a06v21ns.pdf.

13- Lima GO, Cruz RFA, Vale SRM, Carneiro ECRL. Metodologia para implantação de um sistema de gestão da qualidade em um centro de dialise. Relato de experiência. Acta Paul Enferm. 2009 [acesso em: 25 jun. 2016]; 22 (Especial-Nefrologia): 580-2. Disponível em: http://www.scielo.br/ pdf/ape/v22nspe1/28.pdf.

14- Simões M., Brigido BM, Mazon EMA, Pires MDEFC. Água de diálise: parâmetros físico-químicos na avaliação do desempenho das membranas de osmose reversa. Rev. Inst. Adolfo Lutz (Impr.) [on-line], 2005 [acesso em: 2 jun. 2016]; 64 (2) 173-178. Disponível em: http://189.126.110.61/rialutz/article/viewFile/23418/24267.

15- Buzzo ML, Bugno A., Almodovar AAB, Kira CS, Carvalho MFH, Souza A et al. A importância de programas de monitoramento da qualidade da água para diálise na segurança dos pacientes. Rev Inst Adolfo Lutz. São Paulo, 2010 [acesso em: 5 jun. 2016]; 69 (1): 1-6. Disponível em: file://C:/Users/e-Sus/Downloads/6373-8769-1-SM.pdf.

16- Malik AM, Schiesari LMC. Qualidade na Gestão Local de Serviços e Ações de Saúde. (Série Saúde \& Cidadania), vol. São Paulo: Faculdade de Saúde Pública da Universidade de São Paulo, 1998, p. 11-45. Disponível: file://C:/Users/e-Sus/Downloads/saude-cidadania-vol-03-qualidade-na-gestao-local-de-servicos-e-acoes-de-saude-[443-090212-SES-MT].pdf.

17- Marcatto MISJ, Grau MAF, Müller NCS. Projeto de reativação e implantação do Programa de Monitoramento da Água Tratada para Hemodiálise do Estado de São Paulo, SP, ago. 2009. Bepa 
2010 [acesso em: 23 jun. 2016]; 7 (74): 6-12. Disponível em: http://periodicos.ses.sp.bvs.br/pdf/ bepa/v7n74/v7n74a02.pdf

18- Nicole AG, Tronchin DMR. Indicadores para avaliação do acesso vascular de usuários em hemodiálise. Revista Escola de Enfermagem da Universidade de São Paulo 2011; 45 (1): 206-14. Disponível em: http://www.scielo.br/pdf/reeusp/v45n1/29.pdf.

19- Rosetti KAG, Tronchin DMR. Avaliação da conformidade da prática assistencial de manutenção do cateter temporário duplo lúmen para hemodiálise. São Paulo, Rev Esc Enferm USP 2011 [acesso em: 7 jun. 2016]; 45 (1): 206-14. Disponível em: http://www.scielo.br/pdf/rlae/v22n1/ pt_0104-1169-rlae-22-01-00129.pdf

20- Castro MCM, Fatima CS, Xagoraris M, Ruiz JC, Souza JACS. Avaliação do Desempenho Operacional de Dialisadores de Fibra Oca de Polietersulfona em Condições de usos Múltiplos, São Paulo. J Bras Nefrol 2008 [acesso em: 8 jun. 2016]; 30 (22): 144-50. Disponível em: http://www. jbn.org.br/export-pdf/74/30-02-12.pdf.

21- De Oliveira ICP. A legislação sanitária e a qualidade da água nas clínicas de hemodiálise de Salvador. Revista Baiana de Saúde Pública 2014 [acesso em: 28 jun. 2016]; 29: 57. Disponível em: http://rbsp.sesab.ba.gov.br/index.php/rbsp/article/view/1222.

22- De Souza MRG, Silva AEB, Bezerra ALQ, Freitas JS, Miasso AI. Eventos Adversos em Hemodiálise: Relatos de Profissionais de Enfermagem. Rev Esc Enferm USP 2013 [acesso em: 28 jun. 2016]; 47 (1): 75-82. Disponível em: http://www.redalyc.org/pdf/3610/361033324010.pdf.

23- NascimentoVPC, Abud ACF, Inagaki ADM, Daltron AST, Viana LC. Avaliação da técnica de curativo em cliente com acesso venoso para hemodiálise. Revista Enfermagem UERJ. Rev. Enferm 2009 [acesso em: 28 jun. 2016]; 17 (2): 215. http://www.facenf.uerj.br/v17n2/v17n2a13.pdf.

24- Brasil. Portaria n⿳0 2.042 de 11 de outubro de 1996, Gabinete do Ministro - Ministério da Saúde. Diário Oficial da União no. 99, Brasília, de 14/10/96 [acesso em: 23 jul. 2017].

25- Calheiros HC, Guilherme GS. Estudo da potencialidade de reuso da água descartada em sistema de purificação de água usada em tratamentos por hemodiálise. Revista Brasileira de Engenharia Biomédica 2010 [acesso em: 20 out. 2017]; 26 (3): 209-17. Disponível em: http://www.hospitaissaudaveis.org/arquivos/Reaproveitamento\%20da\%20água\%20da\%20hemodiálise.pdf.

26- Morais AS, Melleiro MM. A qualidade da assistência de enfermagem em uma unidade de emergência: a percepção do usuário. Rev. Eletr. Enf. [On-line] 2013 [acesso em: 23 fev. 2017]; 15 (1): 112-20. Disponível em: https://www.fen.ufg.br/revista/v15/n1/pdf/v15n1a13.pdf.

27- Brasil. ANVISA. Agência Nacional de Vigilância Sanitária. RESOLUÇÃO-RDC No. 154, DE 15 DE JUNHO DE 2004. Ministério da Saúde, Brasília, DF, Brasil, 2004 [acesso em: 7 out. 2017]. Disponível em: http://www.saude.mt.gov.br/upload/controle-infeccoes/pasta9/resolucao_rdc_ n154_2004_regulamento_servicos_dialise.pdf.

28- Gama ZAS, Saturno PJ. A segurança do paciente inserida na gestão da qualidade dos serviços de saúde. In: Assistência Segura: Uma Reflexão Teórica Aplicada à Prática. Agência Nacional de Vigilância Sanitária - ANVISA, 2013 [acesso em: 28 jun. 2016], p. 29-40. Disponível em: http:// www.um.es/calidadsalud/archivos/capitulo-\%20Assistencia\%20Segura.pdf.

29- Pertence PP, Melleiro MM. Implantação de ferramenta de gestão de qualidade em Hospital Universitário. Rev Esc Enferm USP 2010 [acesso em: 2 nov. 2017]; 44 (4): 1.024-31. Disponível em: http://www.scielo.br/pdf/reeusp/v44n4/24.pdf.

30- Cavalcante FA, Saar QG, Ramos LS, Lima AAM. O uso do lúdico em hemodiálise: buscando novas perspectivas na qualidade de atendimento ao paciente no centro de diálise. Revista Eletrônica da Facimed 2011 [acesso em: 28 jun. 2017]; 3 (3): 371-84. Disponível em: http://www. facimed.edu.br/o/revista/pdfs/7fbce1022888ed00b20b880492ae5ca0.pdf. 
31- Marisco NS. Novas possibilidades de humanização na hemodiálise: o cliente e a equipe de enfermagem na construção do mais-ser [Dissertação]. Florianópolis (SC): Universidade Federal de Santa Catarina, 2013. Disponível em: https://repositorio.ufsc.br/xmlui/bitstream/handle/123456789/82394/192267.pdf?sequence=1\&isAllowed=y.

32- Brasil. Manual de Padronização de Atos Administrativos Normativos. Brasil. Congresso. Senado. Brasília: 2012 [acesso em: 2 jun. 2017]. Disponível em: https://www2.senado.leg.br/bdsf/ bitstream/handle/id/496338/000960587.pdf? sequence $=1$.

33- Brasil. PORTARIA No. 82, DE 3 DE JANEIRO DE 2000. Ministério da Saúde, Brasília, DF, Brasil. 2000 [acesso em: 7 out. 2017]. Disponível em: http://linus.husm.ufsm.br/janela/legislacoes/ nefrologia/alta-complexidade-em nefrologia/portaria-no-82-de-3-de-janeiro-de-2000.pdf.

34- Brasil. ANVISA. Agência Nacional de Vigilância Sanitária. Resolução RDC no. 11 de 13 de março de 2014. Ministério da Saúde, Brasília, DF, Brasil, 2014 [acesso em: 7 out. 2017]. Disponível em: https://www20.anvisa.gov.br/segurancadopaciente/index.php/legislacao/item/rdc-154de-15-de-junho-de-2004.

\section{DADO(S) AUTOR(RES)}

\section{Darlene Santos Almeida}

Residente em Nefrologia pela Universidade do Estado da Bahia. Enfermeira pelo Centro Universitário Estácio FIB da Bahia. Universidade do Estado da Bahia (UNEB), Salvador/BH - Brasil. darlene. santos.almeida@hotmail.com

\section{Edenise Maria Santos da Silva Batalha}

Enfermeira, Doutoranda da Escola de Enfermagem da Universidade de São Paulo. Professora Auxiliar do Departamento de Ciências da Vida da Universidade do Estado da Bahia (UNEB), Salvador/ BH - Brasil. edenisemaria@gmail.com

Submissão em: 28/11/2017

Aceito em: 11/01/2018 